\title{
25 Research Soure \\ False Safety Behavior Elimination Therapy for Social Anxiety Disorder in Japanese: A Pilot, Single-arm Uncontrolled Study
}

Honami Arai ( $\sim$ honamy821@gmail.com )

Sho Okawa

Yoichi Seki

Eiji Shimizu

Sho Takahashi

Kristina Jo Korte

Norman Brad Schmidt

Research Article

Keywords: social anxiety disorder, safety behavior, anxiety disorder, uncontrolled trial

Posted Date: November 16th, 2020

DOI: https://doi.org/10.21203/rs.3.rs-107578/v1

License: (9) This work is licensed under a Creative Commons Attribution 4.0 International License.

Read Full License 
Running Head: A Pilot study of F-SET for Japanese SAD

1 False Safety Behavior Elimination Therapy for Social Anxiety Disorder in Japanese:

A Pilot, Single-arm Uncontrolled Study

3

4 Abstract

5 Social anxiety disorder (SAD) is an excessive fear of social situations that can lead to

6 serious functional impairment; it negatively affects a person's self-esteem and social

7 interactions. Its prevalence has increased over the past four decades, and SAD is one of

8 the most prevalent psychiatric disorders in Japan. Therefore, more precise interventions

9 are needed. This study administered false safety behavior elimination therapy (F-SET),

10 which eliminates safety behavior (i.e., strategies that reduce anxiety), to six Japanese

11 patients with SAD. This is the first adaptation of F-SET into Japanese. Seven patients

12 were screened, 6 (mean age of 27.83 , only males) were eligible for the study criteria.

13 All patients received five weekly 60-minute F-SET sessions. At post-F-SET, the mean

14 reduction in Liebowiz Social Anxiety Scale (LSAS) scores from baseline was -20.50

15 (95\%CI: $-30.65,-10.35, p=.003)$. After completing the treatment, all participants

16 showed substantially decreased SAD symptoms, which indicates preliminary support

17 for the adapted treatment's efficacy.

18

19 Keywords: social anxiety disorder, safety behavior, anxiety disorder, uncontrolled trial. 
1 Social anxiety disorder (SAD) is an anxiety disorder that is characterized by fear or

2 anxiety in social situations. SAD can negatively interfere with a person's social life and

3 academic performance. One epidemiological study suggests that approximately $25 \%$ of

4 the population will suffer from clinically significant social anxiety at some point, with a

5 12-month prevalence of about 16\% (Bandelow \& Michaelis, 2015). To address the need

6 for psychological interventions for social anxiety psychopathology, there has been an

7 increased interest in the use of cognitive behavioral therapies (CBT; Barlow, 2002;

8 Norton \& Paulus, 2016). Studies have shown that CBT has consistently been effective

9 in reducing social anxiety in randomized controlled trials (RCTs; Mayo-Wilson et al.,

10 2014). It has longer-lasting effects, fewer adverse reactions, lower relapse rates, and

11 greater acceptability in the field (Gelernter, Uhde, Climbolic, Arnkoff, Vittone, Tancer,

12 \& Bartko., 1991; Heimberg et al., 1998; Hofmann et al., 1998) than other therapies.

13 However, there has been a demand for using brief CBT interventions as a part of

14 stepped care. Briefer treatment may be more cost-effective, which can increase

15 treatment accessibility. A promising approach to increase access to evidence-based

16 therapy for SAD is a brief and simple intervention named the False Safety Behavior

17 Elimination Therapy (F-SET), which may be easier for patients to attend.

Safety behaviors are coping strategies that temporarily alleviate anxiety

19 (Schmidt, Buckner, Pusser, Woolaway-Bickel, Preston, \& Norr, 2012). For example,

20 people with social anxiety may take slow breaths and grip objects tightly to avoid

21 shaking while reading to a group, speak quickly or rehearse sentences in their mind to

22 counter the fear of talking funny, or avoid eye contact to deal with the fear of freezing

23 up while making conversation (Wells et al., 2016. Safety behaviors are utilized with the

24 intention of making the individual feel "safe" in the context of their phobic anxiety.

25 While these behaviors can provide temporary relief, in the long-term they can maintain 
1 and exacerbate anxiety (Salkovskis, 1991). This is because once individuals begin to

2 use safety behaviors (e.g., avoidance), they often attribute the reduction in anxiety to

3 their safety behaviors and view them as effective coping strategies. In the

4 developmental trajectory, the expansion of safety behaviors and anxiety can create a

5 negative feedback loop as one contributes to the other. The utilization of safety behavior

6 appears to have a detrimental impact on the efficacy of exposure therapy, which is one

7 of the most effective treatments for anxiety disorders. For example, Kim (2005) found

8 that SAD involving cognitive rationale (safety behavior) resulted in lower fear reduction

9 during exposure among individuals when compared to SAD involving instructions to

10 drop safety behavior. Similarly, Morgan and Raffle (1999) found that individuals with

11 SAD fears showed greater fear reduction when instructed to drop safety behaviors than

12 in the case of exposure plus safety behavior utilization. In essence, these studies have

13 reported that eliminating safety behaviors enhanced outcomes. Thus, treatment targeting

14 safety behavior may offer a simplified approach to dealing with anxiety problems.

15 Schmidt and colleagues (Riccardi, Korte, \& Schmidt, 2017; Schmidt et al.,

16 2012) developed F-SET to create a simple treatment for anxiety disorders through the

17 identification and elimination of safety behaviors. A major strength of F-SET is its

18 straightforward nature. It identifies and eliminates safety behaviors that tend to be

19 shared across anxiety disorders. Many CBT protocols for anxiety include education,

20 cognitive reappraisal, and exposure exercises. In F-SET, patients receive general

21 psychoeducation, though training in cognitive therapy and exposure are not specifically

22 covered. Nonetheless, the focus on eliminating safety aids and developing an anti-

23 phobic approach commonly leads patients to complete exposure exercises during the

24 course of the treatment (Schmidt et al., 2012). Both individual and group F-SET instruments have been developed. The efficacy of F-SET has been evaluated in several 
1 RCTs. In the first clinical trial, Schmidt et al. (2012) examined its effectiveness in

2 mixed groups of patients with $\mathrm{SAD}$, panic disorder, and generalized anxiety disorder.

3 Riccardi et al. (2017) followed this study by examining the efficacy of the F-SET

4 version designed for individuals, with a 5-week individual therapy format. The

5 individual F-SET format allowed for shorter treatment duration since the therapist

6 could focus on the safety aids used most frequently by the client, rather than the broader

7 range of safety aids covered in the group format (Riccardi et al., 2017). Both studies

8 found a significant reduction in anxiety and depression symptoms immediately post-

9 treatment and at the one-month follow-up appointment, when compared to the wait-list

10 control group.

11 However, the studies providing evidence for F-SET's efficacy in several trials

12 were conducted in the United States. Thus, the generalizability of F-SET to other

13 cultural contexts is unknown. As such, we are currently conducting a stringent RCT of

14 F-SET for SAD. The goal of this controlled trial is to test the efficacy of F-SET on

15 SAD. Examinations of the efficacy of a brief intervention of F-SET for SAD as well as

16 in non-Western populations are both first reported attempts in the field of clinical

17 psychology. Since previous studies have reported that CBT developed for patients in

18 Western culture is effective for Japanese patients as well (e.g., Yoshinaga et al., 2013),

19 we hypothesize that F-SET will have a similar effect on a Japanese sample as that found

20 for participants in previous studies conducted in Western countries However, it can be

21 assumed Japanese individuals are less willing to seek treatment than Western

22 individuals. A previous study has found the Japanese to be reluctant to seek professional

23 help compared to Americans (Mojaverian et al., 2013). Also, Asians prefer implicit

24 social support like talking to their family while European Americans prefer explicit

25 social support like seeking professional help (Taylor et al., 2007). Hence, it is important 
1 to examine the feasibility such as the recruitment rate and acceptability of F-SET before

2 conducting an RCT for a large number of patients. The present study adapted the F-SET

3 for a Japanese population and presented its feasibility, safety, and preliminary efficacy

4 under conditions of the F-SET being administered individually to patients with SAD.

5 Given the scarcity of practitioners in Japan, the F-SET could represent an efficient

6 alternative to other CBT protocols for anxiety disorders; it requires less time and is

7 based on a straightforward theoretical background. Its use may be more acceptable and

8 feasible in areas facing practitioner shortages. A pilot study is defined as a stand-alone

9 piece of work planned and carried out independently to the main study (Lancaster,

10 Dodd, \& Williamson, 2004). At the first stage of the RCT, this study assessed the

11 feasibility (i.e., recruitment rate), acceptability (i.e., treatment adherence and dropout

12 rate), safety (i.e., occurrence of adverse events [AEs]), and a preliminary estimate of the

13 F-SET's potential utility in Japanese clinical settings.

\section{Methods}

Participants. This study was a single-arm, pilot study conducted at Chiba

17 University in Japan. As this study was aiming to assess feasibility, no formal sample size calculation was undertaken. The criteria for inclusion were a primary

19 diagnosis of SAD according to the Diagnostic and Statistical Manual of Mental

20 Disorders, $5^{\text {th }}$ edition (DSM-5), and an age of 18-65 years. All patients had been

21 referred by psychiatric clinics to our Cognitive Behavioral Therapy Center in

22 Chiba University to reduce their social anxiety problems. We set a cutoff score of

2330 on the Liebowitz Social Anxiety Scale (LSAS) for screening patients suffering

24 from SAD symptoms. This score was based on the remission score of SAD in

25 previous study (Pollack et al., 2014). Comorbid diagnoses were permitted if they 
1 were clearly secondary (i.e., the SAD symptoms were more severe and

2 impairing). The exclusion criteria included presence of psychosis, pervasive

3 developmental disorders/intellectual disability, autism spectrum disorder (an

4 Autism Spectrum Quotient score $\geq 32$ ), a current high risk of suicide, substance

5 abuse or dependence in the past six months, anti-social personality disorder, or an

6 unstable medical condition. The baseline assessments, and the 5-week post-

7 treatment and 9-week follow-up (FU) assessments were completed in the

8 Cognitive Behavioral Therapy Center at Chiba University. Clinical interviews

9 were conducted by a doctoral student in clinical psychology.

Outcomes to assess feasibility. (a) Screening and eligibility, the screening rate

11 was defined as the number of patients who had contact with the recruitment team and

12 who were assessed for eligibility using inclusion and exclusion criteria. This included

13 those who decided not to take part in the study. Eligibility was determined by dividing

14 the number of people screened by the number of people who met the inclusion criteria.

15 (b) Adverse events, For the safety analysis, frequencies and proportions of AEs were

16 summarized. (c) Retention rate, the retention rate was defined as the number of

17 participants who remained in the study and did not drop out. (d) Completion rate, the completion rate was defined as the number of participants who completed the target

19 number of five F-SET sessions. (e) Intervention adherence, Intervention adherence was 20 calculated by summing the total number of participants and the target number of F-SET sessions (5 per participant) and comparing this against the actual completed sessions.

Outcome measures to assess efficacy. The primary outcome measured was the LSAS change between the baseline and 5-week scores. The LSAS (Liebowitz, 1987) is

24 one of the most commonly used clinician-administered scales for measuring social phobia. The Japanese-language LSAS has good reliability and validity (Asakura et al., 
Running Head: A Pilot study of F-SET for Japanese SAD

1 2002). The questionnaire also assessed patient's depressive symptoms using the Beck

2 Depression Inventory (BDI-II; Beck et al., 1996), social disability using the Sheehan

3 Disability Scale (SDS; Sheehan, Harnett-Sheehan, \& Raj, 1983), and the frequency of

4 safety behavior using the Subtle Avoidance Frequency Examination (SAFE; Cuming,

5 Rapee, Kemp, Abbott, Peters, \& Gaston., 2009). AEs were assessed throughout the trial.

6 Participants were compensated for the time taken to complete the post-intervention

7 assessments with a Japanese $¥ 4,000$ (US\$37) gift voucher.

Therapist and quality control. All sessions were delivered by the first author, who is a clinical psychologist trained in using CBT to treat anxiety disorders. The first author was trained on the F-SET protocol by the last author and the work was thoroughly monitored by a psychiatrist during weekly supervised sessions. The therapist was trained via a 2-hour in-person workshop that included didactic instruction on anxiety problems, training in the F-SET protocol, and discussion of case examples. To support treatment fidelity, a therapist manual was scripted and included structured worksheets to deliver skills training.

Manual translation process. We developed a Japanese version of the F-SET manual that was semantically equivalent to the original one (Riccardi et al., 2017) by a translation/back-translation procedure. After the process, the authors of the original manual compared the original and back- translated versions and confirmed that the two versions were essentially equivalent.

Statistical analysis. The analyses of the primary and secondary efficacy outcomes were performed using the Full Analysis Set. Safety analysis was conducted in the safety analysis population. For baseline characteristics, summary statistics included frequencies and proportions for categorical variables, and means and standard deviations $(S D s)$ for continuous variables. 
In the primary analysis, to evaluate potential treatment efficacy, the mean difference in the change in LSAS between the baseline and 5-week post appointments and its 95\% confidence interval (CI) was estimated using a Wilcoxon signed-rank test. We calculated effect sizes using the formula for Cohen's $d$ for pre- to 5-week posttreatment LSAS score.

For the secondary analysis, the means and their $95 \%$ CIs were estimated for continuous variables, frequencies, and proportions for the categorical variables. Since this was a pilot study and no sample size or power calculation was performed on outcome measures, all comparisons were considered exploratory in nature, regardless of the significance level found.

Compliance with Ethical Standards. This study was conducted in the psychiatric outpatient section of the Chiba University Hospital and performed in compliance with the Declaration of Helsinki principles. The study protocol was approved by the Ethics Committee of Chiba University Hospital (reference number: G29058), and is registered in the national UMIN Clinical Trial Registry (reference number: UMIN000032064). All participants provided written informed consent before participating in the study.

\section{Results}

Screening eligibility. A total of seven participants were screened. Figure 1 shows the flow of participants throughout the trial. Of the seven patients identified, one (86\%) was not eligible to partake in the trial, as this participant no longer met the diagnostic criteria for SAD since their original diagnosis. The remaining six patients received information about the trial. 
1 enrolled in the study (Table 1). All participants were male, and the patients' mean age

2 was $28.30(S D=11.6)$ years. All participants met the principal DSM-5 diagnostic

3 criteria for SAD (mean duration of illness: 10.0 years). Additional Axis I diagnosis for

4 patients included major depressive disorder (33\%). Furthermore, three participants

$5 \quad(50.0 \%)$ were taking psychotropic medication [Benzodiazepines $(n=1)$;

6 Antidepressants $(n=1)$; both Benzodiazepine and Antidepressants $(n=1)]$. There were

7 no changes in pharmacotherapy during the intervention.

Outcome measures for feasibility. There were no AEs reported during or

9 because of the F-SET intervention. The retention rate was $100 \%$ for the patients who consented to partake in the study. The completion rate was based on the number of

11 participants who completed the target number of five sessions. Of the six participants

12 who enrolled in the study, all participants managed all sessions. Intervention adherence was calculated by summing the total number of participants and the target number of FSET sessions and comparing this against the actual completed sessions. Therefore, the maximum number of sessions possible was 30 (6 participants who consented to partake $\times 5)$. As such, the completion rate was $100 \%$.

Outcome measures for efficacy. Figure 2 presents the change in social anxiety symptoms from the baseline assessment to the 9-week FU assessment. In the primary analyses to examine the LSAS change score from pre- to post-treatment, a significant

20 difference $(p=.0031)$ was found. The reduction of LSAS was maintained at the 9-week follow-up assessment [-22.83 (95\%CI: -39.39, -6.27)]. For pre to post, the effect size for LSAS was large (Cohen's $d=0.83$ ). Table 2 shows the individual changes of all

23 outcome measures such as the LSAS, BDI-II, SDS, and SAFE. The mean total scores of 24 the BDI-II decreased from the baseline to 5-week post-treatment [-8.00 (95\% CI: 15.68, -0.32)], and 9-week FU treatment [-14.67 (95\% CI: -23.97, -5.36)]. The mean 
Running Head: A Pilot study of F-SET for Japanese SAD

1 total scores of the SDS decreased from the baseline to 5-week post treatment [-4.17

2 (95\%CI: -10.44, 2.11)], and 9-week FU treatment [-7.67 (95\% CI: -14.39, -0.94)]. The

3 mean total scores of the SAFE decreased from the baseline to 5-week post-treatment [-

46.33 (95\%CI: -16.78, 4.11)], and 9-week FU treatment [-11.00 (95\% CI: -26.48, 4.48)].

5

6 Discussion

7 The F-SET protocol is an intervention designed to identify and eliminate safety

8 behaviors. This small pilot trial in Japan demonstrates the feasibility, safety, and

9 preliminary efficacy of the brief 5-session F-SET treatment protocol among a sample of

10 Japanese with SAD. The key observations from this initial pilot study were: (a) the

11 intervention is administrable in Japanese clinical settings; (b) it was generally well

12 received by social anxiety patients with no AEs; and (c) it seemed to reduce social

13 anxiety for most, but not all patients.

No severe AE was observed during the sessions. Findings were also consistent

with an intervention showing good acceptability based on dropout rates. The negligible dropout rate in this trial was consistent with the dropout rate in other F-SET trials (19$26 \%)$ as well as in traditional CBT protocols for anxiety disorders (5.6-19.0\%) (Otto, Smits, \& Reese, 2004). These results speak to the viability of F-SET in medical settings in Japan. In addition, our results of a high completion rate and intervention adherence with very low patient attrition reflect that the F-SET intervention was well tolerated.

21 Generalizability is another key element of clinical utility. Of course, generalizability is a central design element for any unified treatment. In terms of effectiveness, our F-SET program also compares favorably with

24 Western reports. For example, several previous meta-analyses of CBT for SAD have derived effect sizes from pre- to post-treatment between 0.62 and 1.27 for those 
1 completing the treatment (Otte, 2011). Hence, it can be assumed that F-SET programs

2 have a tendency toward large effect size, similar to traditional CBT. However, our

3 effect size of 0.83 in terms of clinician administered LSAS scores between the pre- and

4 post-F-SET observation was smaller relative to the effect size of 1.56

${ }^{1}$ found in previous clinical trials on CBT protocol in Japan that used the model

6 of Clark and Wells (Yoshinaga et al., 2013). However, Clark and Wells' CBT protocol

7 takes considerably more time and cost to participate for both the patients and therapist.

8 Thus, the F-SET treatment can be an option for patients having a low severity of SAD,

9 and for introducing a new treatment in institutions unfamiliar with evidence-based

10 treatments. For example, people with full-time jobs or those busy with academics may

11 find it difficult to take off time during the work week for weekly appointments;

12 however, it may be possible for them to commit to a weekend of intensive treatment.

13 Stepped care interventions, where traditional CBT is offered before F-SET to those with

14 more severe symptoms, or to those whose symptoms do not remit following the F-SET,

15 could capitalize on the benefits of both treatment formats to provide optimal gains at

16 less cost.

The result of our study indicated that F-SET contributes to reducing social anxiety symptoms by eliminating safety behavior, which plays a central role in maintaining anxiety symptoms with a large effect size. However, this study employed a

20 small sample size, and the mean score could have been influenced by a few patients;

21 hence, we looked further into the change in the symptoms of each patient. This initial

22 pilot study showed that the intervention seemed to reduce social anxiety for most, but

23 not all patients. According to Table 2, though all patient anxiety symptoms decreased

24 from pre- to post-treatment, two patients' (A and E) social anxiety returned from posttreatment to FU. In addition, patients' A and E's safety behavior scores did not change 
1 throughout the course. These combined results suggest that most SAD patients do

2 benefit from the F-SET, but all patients may not. The reason behind this disadvantage

3 faced by the two patients in our study could be their inability to generalize the acquired

4 CBT skills in their daily life setting, as each component in our CBT protocol took place

5 in only one session over the five weeks. This is because some SAD patient with rigid

6 cognitive biases might find it difficult to eliminate their safety behavior due to their firm

7 belief of experiencing a negative evaluation in social settings. This reasoning implies

8 that traditional CBT is more suitable for some patients than F-SET. Further examination

9 of the characteristics of patients that influence the suitability of F-SET for them is 10 warranted.

11 Of the six patients in this study, three met the criteria for major depressive

12 disorder. In routine clinical settings, there are many patients with such comorbidities

13 (Koyuncu, İnce, Ertekin, \& Tükel, 2019). It should be noted that depressive symptoms

14 also reduced for all those participating in this study. The depression levels of all the six

15 patients were altered in an adaptive direction at one week and one month after the F-

16 SET intervention. These findings are consistent with RCTs conducted in the United

17 States that showed that F-SET can affect mood psychopathology (e.g., Schmidt et al., 18 2012).

20 Limitations and Future Directions

21 Overall, although our present study provides highly valuable information, it does have some limitations including its small sample size, all male participants, no control group,

23 and no long-term follow-up data. Despite these limitations, our results suggest that F-

24 SET is a feasible, potentially effective treatment for SAD in Japanese clinical settings.

25 Further research is needed to address the limitations of this study, such as RCTs with 
Running Head: A Pilot study of F-SET for Japanese SAD

1 control groups.

2

3

\section{Conflict of Interest}

4

5

The authors declare no conflicts of interest associated with this manuscript.

6 
Running Head: A Pilot study of F-SET for Japanese SAD

\section{References}

American Psychiatric Association. (2013). Diagnostic and statistical manual of mental disorders (5th ed.). Washington, D.C: American Psychiatric Association.

Asakura, S., Inoue, S., Sasaki, F., Sasaki, Y., Kitagawa, N., Inoue, T., ...Koyama, T. (2002). Reliability and validity of the Japanese version of the Liebowitz social anxiety scale. Japanese Journal of Psychiatric Medicine, 44, 1077-1084. (In Japanese with English abstract.)

Bandelow, B., \& Michaelis, S. (2015). Epidemiology of anxiety disorders in the 21st century. Dialogues in Clinical Neuroscience, 17(3), 327-335.

Barlow, D. H. (2002). Anxiety and its disorders: The nature and treatment of anxiety and panic (2nd ed.). New York, NY: Guilford Press.

Beck, A.T., Steer, R. A., \& Brown, G. K. (1996). Manual for the Beck Depression Inventory-II (BDI-II). San Antonio, TX: Psychological Corporation.

Cuming, S., Rapee, R. M., Kemp, N., Abbott, M. J., Peters, L., \& Gaston, J. E. (2009). A self-report measure of subtle avoidance and safety behaviors relevant to social anxiety: Development and psychometric properties. Journal of Anxiety Disorders, 23, 879-883. https://doi.org/10.1016/j.janxdis.2009.05.002

Gelernter, C. S., Uhde, T. W., Cimbolic, P., Arnkoff, D. B., Vittone, B. J., Tancer, M. E., \& Bartko, J. J. (1991). Cognitive-behavioral and pharmacological treatments of social phobia. A controlled study. Archives of General Psychiatry, 48(10), 938945. https://doi.org/10.1001/archpsyc.1991.01810340070009

Heimberg, R. G., Liebowitz, M. R., Hope, D. A., Schneier, F. R., Holt, C. S., Welkowitz, L. A., ...Klein, D. F. (1998). Cognitive behavioral group therapy vs phenelzine therapy for social phobia: 12-week outcome. Archives of General Psychiatry, 55(12), 1133-1141. https://doi.org/10.1001/archpsyc.55.12.1133 
Running Head: A Pilot study of F-SET for Japanese SAD

1 Heimberg, R. G., Stein, M. B., Hiripi, E., \& Kessler, R. C. (2000). Trends in the prevalence of social phobia in the United States: A synthetic cohort analysis of changes over four decades. European Psychiatry, 15(1), 29-37.

Hofmann, S. G., Barlow, D. H., Papp, L. A., Detweiler, M. F., Ray, S. E., Shear, M. K., ...Gorman, J. M. (1998). Pretreatment attrition in a comparative treatment outcome study on panic disorder. American Journal of Psychiatry, 155(1), 43-47.

Kim, E. J. (2005). The effect of the decreased safety behaviors on anxiety and negative thoughts in social phobics. Journal of Anxiety Disorders, 19(1), 69-86. https://doi.org/10.1016/j.janxdis.2003.11.002

Koyuncu, A., İnce, E., Ertekin, E., \& Tükel, R. (2019). Comorbidity in social anxiety disorder: Diagnostic and therapeutic challenges. Drugs in Context, 8, 212573-13. https://doi.org/10.7573/dic. 212573

Lancaster, G. A., Dodd, S., \& Williamson, P. R. (2004). Design and analysis of pilot studies: recommendations for good practice. Journal of Evaluation in Clinical Practice, 10(2), 307-312. https://doi.org/10.1111/j..2002.384.doc.x

Liebowitz, M. R. (1987). Social phobia. Modern Problems in Pharmacopsychiatry, 22, 141-173. https:// https://doi.org/10.1159/000414022.

Mayo-Wilson, E., Dias, S., Mavranezouli, I., Kew, K., Clark, D. M., Ades, A. E., \& Pilling, S. (2014). Psychological and pharmacological interventions for social anxiety disorder in adults: A systematic review and network meta-analysis. Lancet Psychiatry, 1, 368-376.

Mojaverian, T., Hashimoto, T., \& Kim, H. S. (2013). Cultural differences in professional help seeking: a comparison of Japan and the U.S. Frontiers in Psychology, 3, 615. https://doi.org/10.3389/fpsyg.2012.00615

Morgan, H., \& Raffle, C. (1999). Does reducing safety behaviours improve treatment 
Running Head: A Pilot study of F-SET for Japanese SAD

response in patients with social phobia? Australian and New Zealand Journal of Psychiatry, 33(4), 503-510. https://doi.org/10.1080/j.1440-1614.1999.00599.x

Norton, P. J., \& Paulus, D. J. (2016). Toward a unified treatment for emotional disorders: Update on the science and practice. Behavior Therapy, 47(6), 854-868.

Otte, C. (2011). Cognitive behavioral therapy in anxiety disorders: Current state of the evidence. Dialogues in Clinical Neuroscience, 13(4), 413-421.

Otto, M. W., Smits, J. A. J., \& Reese, H. E. (2004). Cognitive-behavioral therapy for the treatment of anxiety disorders. Journal of Clinical Psychiatry, 65 (Suppl 5), 3441.

Pollack, M. H., Van Ameringen, M., Simon, N. M., Worthington, J. W., Hoge, E. A., Keshaviah, A., \& Stein, M. B. (2014). A double-blind randomized controlled trial of augmentation and switch strategies for refractory social anxiety disorder. American Journal of Psychiatry, 171(1), 44-53.

Riccardi, C. J., Korte, K. J., \& Schmidt, N. B. (2017). False safety behavior elimination therapy: A randomized study of a brief individual transdiagnostic treatment for anxiety disorders. Journal of Anxiety Disorders, 46, 35-45.

Salkovskis, P. M. (1991). The importance of behaviour in the maintenance of anxiety and panic: A cognitive account. Behavioural Psychotherapy, 19, 6-19. https://doi.org/10.1017/S0141347300011472

Schmidt, N. B., Buckner, J. D., Pusser, A., Woolaway-Bickel, K., Preston, J. L., \& Norr, A. (2012). Randomized controlled trial of false safety behavior elimination therapy: A unified cognitive behavioral treatment for anxiety psychopathology. Behavior Therapy, 43(3), 518-532.

Sheehan, D., Harnett-Sheehan, K., \& Raj, B. (1996) The measurement of disability. International Clinical Psychopharmacology, 11, 89-95. 
Running Head: A Pilot study of F-SET for Japanese SAD

1 Wells, A., Clark, D. M., Salkovskis, P., Ludgate, J., Hackmann, A., \& Gelder, M. (2016).

2 Social Phobia: The Role of In-Situation Safety Behaviors in Maintaining Anxiety and Negative Beliefs - Republished Article. Behavior Therapy, 47(5), 669-674. https://doi.org/10.1016/j.beth.2016.08.010

Taylor, S. E., Welch, W. T., Kim, H. S., \& Sherman, D. K. (2007). Cultural differences in the impact of social support on psychological and biological stress responses. Psychological Science, 18(9), 831-837. https://doi.org/10.1111/j.1467-9280.2007.01987.x

Yoshinaga, N., Ohshima, F., Matsuki, S., Tanaka, M., Kobayashi, T., Ibuki, H., ... 0 Shimizu, E. (2013). A preliminary study of individual cognitive behavior therapy 1 for social anxiety disorder in Japanese clinical settings: A single-arm, 2 uncontrolled trial. BMC Research Notes, 6(1), 74. https://doi.org/10.1186/1756-0500-6-74 
Running Head: A Pilot study of F-SET for Japanese SAD

${ }^{1}$ To compare the effect size of LSAS in our study with the effect size in the previous study, we calculated Cohens'd for the previous study conducted by Yoshinaga et al. (2013) based on their sample size, mean score for pre and post, and $S D$ for pre and post. 


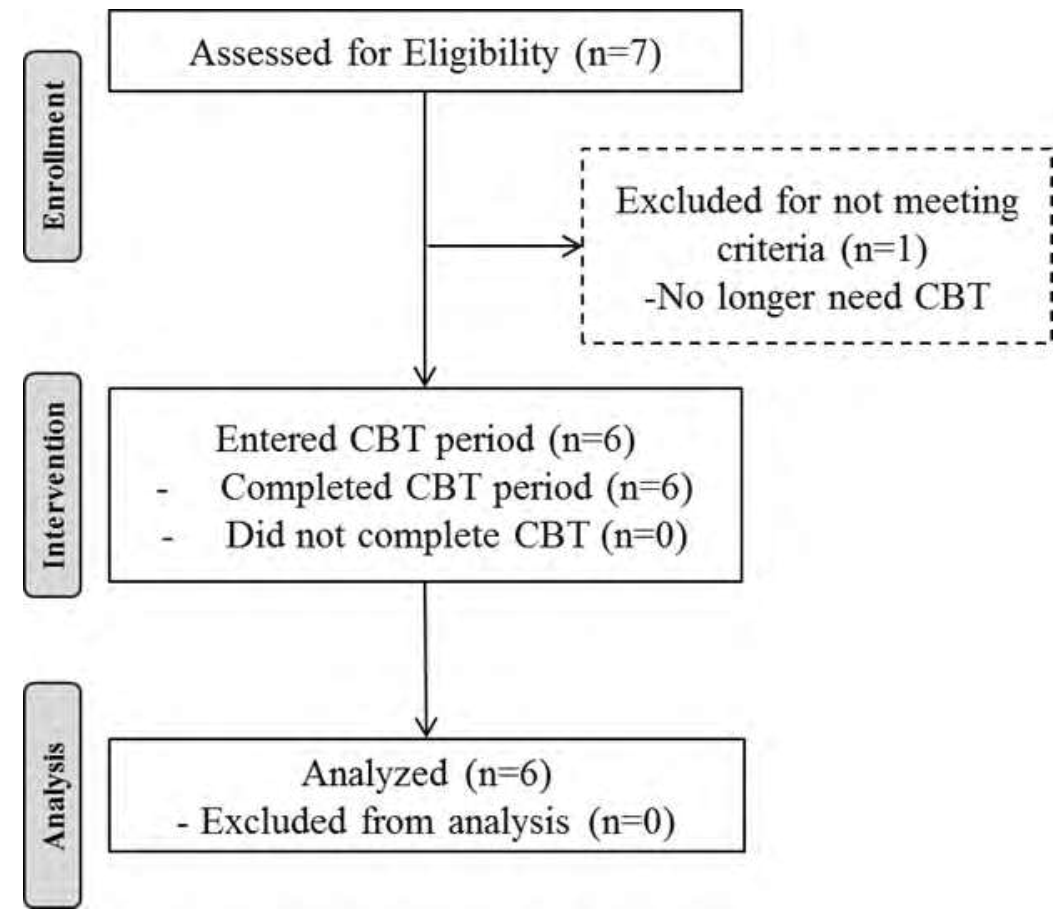

Figure 1. Screening, enrolment, and follow-up of the patients. 


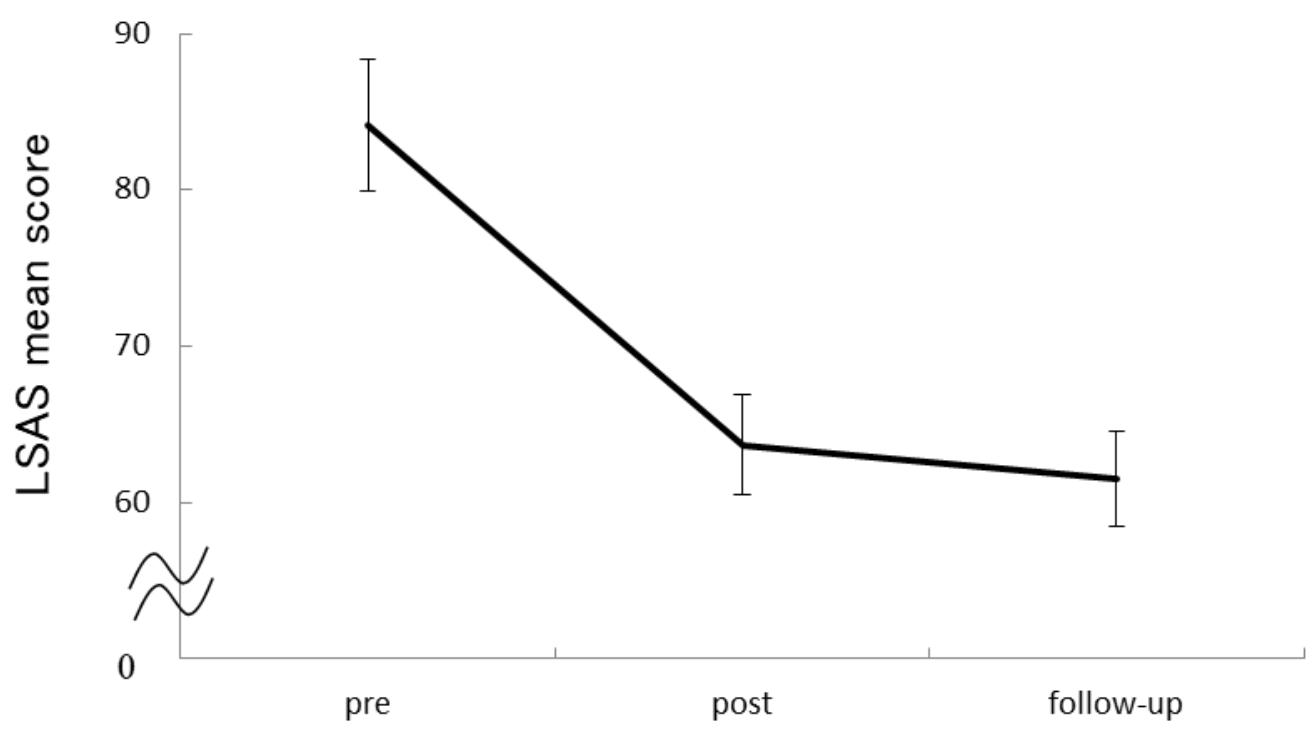

Figure 2. Mean social anxiety severity at each time point. 
Table 1.

Demographic and clinical information of the participants

\begin{tabular}{|c|c|c|}
\hline \multirow{2}{*}{$\begin{array}{l}\text { Characteristic } \\
\text { Age (years), Mean }(S D)\end{array}$} & \multicolumn{2}{|c|}{ Value $(n=6)$} \\
\hline & 28.3 & $(11.6)$ \\
\hline Age of onset (years), Mean $(S D)$ & 18.0 & $(5.45)$ \\
\hline Duration of SAD (years), Mean $(S D)$ & 10.0 & $(9.61)$ \\
\hline \multicolumn{3}{|l|}{ Gender } \\
\hline Male, n (\%) & 6 & $(100)$ \\
\hline \multicolumn{3}{|l|}{ Marital status } \\
\hline Single, n (\%) & 5 & $(83.3)$ \\
\hline Divorced, n (\%) & 1 & $(16.7)$ \\
\hline \multicolumn{3}{|l|}{ Education } \\
\hline Graduate school, n (\%) & 1 & $(16.7)$ \\
\hline University, n (\%) & 2 & $(33.3)$ \\
\hline High school, n (\%) & 3 & $(50.0)$ \\
\hline \multicolumn{3}{|l|}{ Comorbidity } \\
\hline With comorbidity, n (\%) & 3 & $(50.0)$ \\
\hline Without comorbidity, n (\%) & 3 & $(50.0)$ \\
\hline \multicolumn{3}{|l|}{ Medication } \\
\hline Benzodiazepine(BZ), n (\%) & 1 & $(16.7)$ \\
\hline Antidepressant(AD), n (\%) & 1 & $(16.7)$ \\
\hline Both BZ and AD use, n (\%) & 1 & $(16.7)$ \\
\hline No medication, $\mathrm{n}(\%)$ & 3 & $(49.9)$ \\
\hline
\end{tabular}


Table 2.

Outcome measures of six patients.

\begin{tabular}{|c|c|c|c|c|c|c|c|c|c|}
\hline & & \multicolumn{7}{|c|}{ Patient } & \multirow[b]{2}{*}{$(S D)$} \\
\hline & & A & B & $\mathrm{C}$ & D & E & $\mathrm{F}$ & Mean & \\
\hline \multicolumn{10}{|c|}{$\begin{array}{l}\text { Clinical Outcomes: } \\
\text { LSAS }\end{array}$} \\
\hline & Pre & 70 & 87 & 56 & 91 & 85 & 116 & 84.17 & (20.31) \\
\hline & Post & 51 & 58 & 25 & 67 & 70 & 111 & 63.67 & $(28.21)$ \\
\hline & Follow-up & 72 & 57 & 21 & 59 & 77 & 82 & 61.5 & $(22.08)$ \\
\hline
\end{tabular}

BDI- II

$\begin{array}{lcccccccc}\text { Pre } & 12 & 34 & 10 & 26 & 22 & 48 & 25.33 & (13.24) \\ \text { Post } & 6 & 13 & 2 & 23 & 18 & 47 & 18.17 & (16.07) \\ \text { Follow-up } & 8 & 5 & 8 & 8 & 5 & 35 & 11.5 & (11.61)\end{array}$

SDS

$\begin{array}{lcccccccc}\text { Pre } & 5 & 19 & 12 & 26 & 11 & 28 & 16.83 & (8.97) \\ \text { Post } & 5 & 5 & 12 & 17 & 12 & 26 & 12.83 & (7.94) \\ \text { Follow-up } & 3 & 5 & 11 & 9 & 7 & 21 & 9.33 & (6.38)\end{array}$

$\begin{array}{llllllllll}\text { SAFE } & \text { Pre } & 80 & 102 & 61 & 115 & 81 & 126 & 94.17 & (24.42)\end{array}$

$\begin{array}{llllllllll}\text { Post } & 85 & 83 & 49 & 101 & 84 & 125 & 87.83 & \text { (24.94) }\end{array}$

$\begin{array}{lllllllll}\text { Follow-up } & 83 & 72 & 54 & 89 & 87 & 114 & 83.17 & \text { (19.87) }\end{array}$

Note: LSAS= Liebowitz Social Anxiety Scale, BDI- II =Beck Depression Inventory,

SDS $=$ Sheehan Disability Scale, SAFE $=$ Subtle Avoidance Frequency Examination. 
Figures

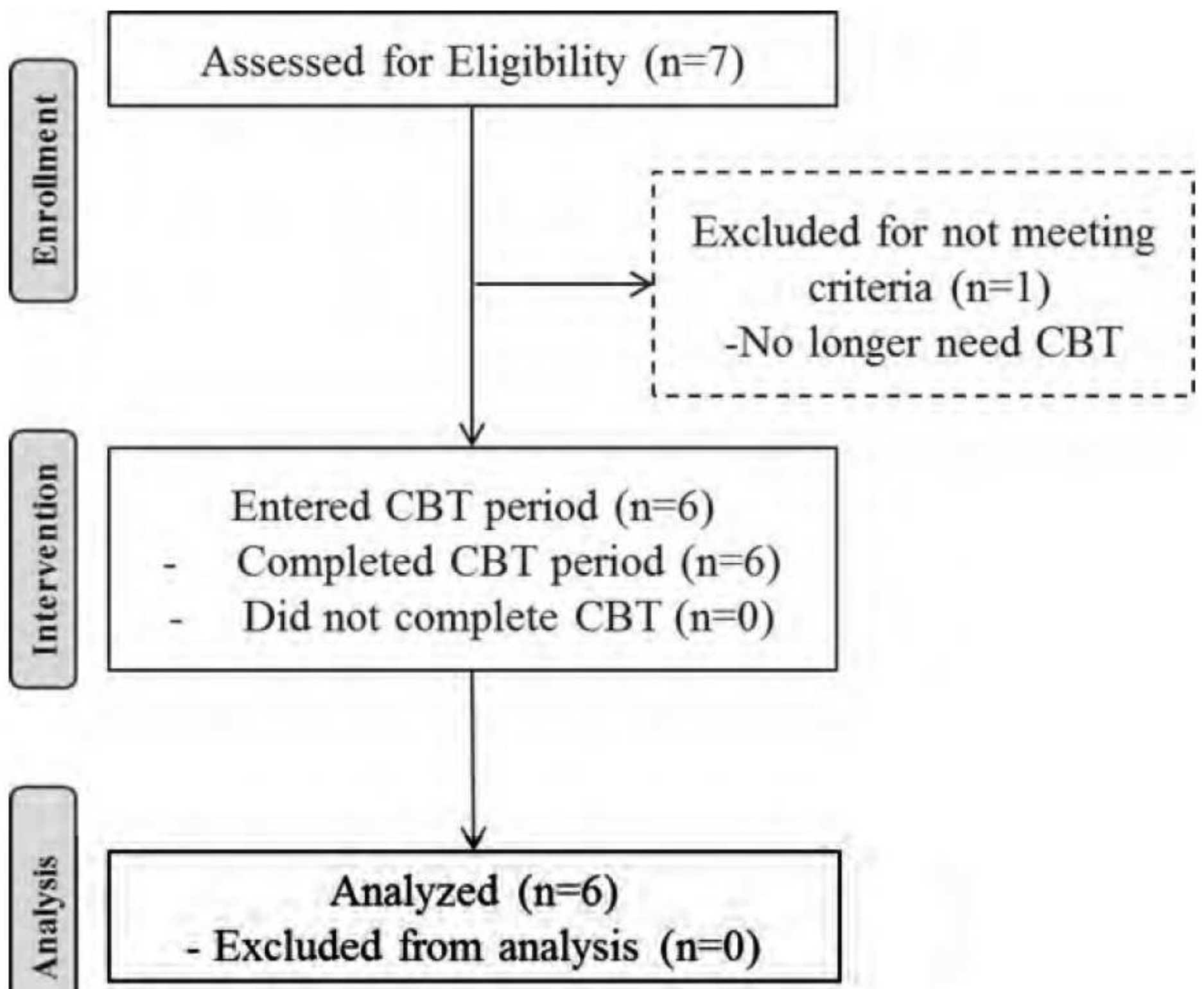

Figure 1

Screening, enrolment, and follow-up of the patients. 


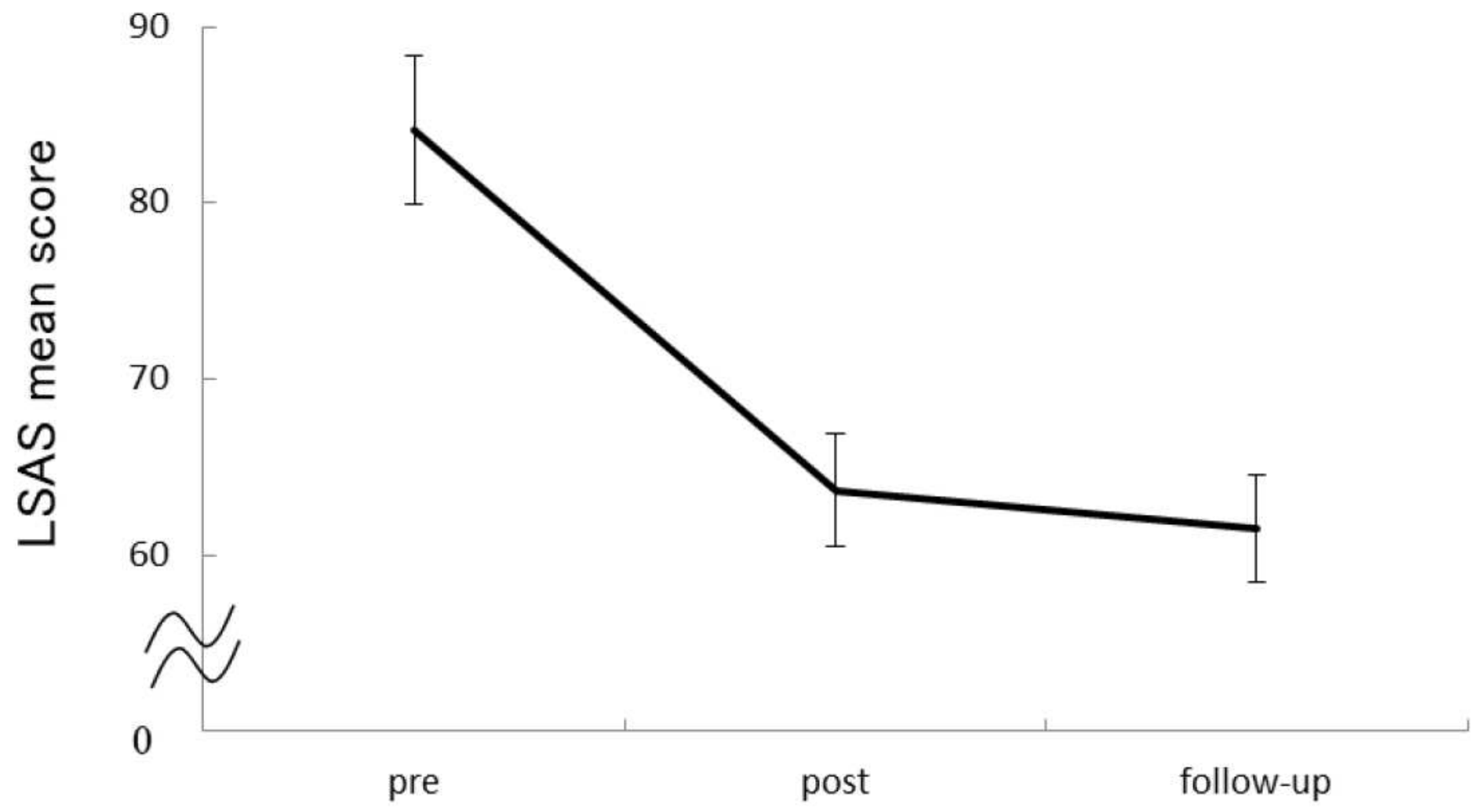

Figure 2

Mean social anxiety severity at each time point. 